\title{
Dielectric properties of solution-grown-undoped and acrylic-acid-doped ethyl cellulose
}

\author{
P K KHARE* and SANDEEP K JAIN ${ }^{\dagger}$ \\ Department of Postgraduate Studies and Research in Physics and Electronics, Rani Durgavati University, \\ Jabalpur 482 001, India \\ †University Science Instrumentation Centre, Dr H S Gour University, Sagar 470 002, India
}

MS received 14 September 1999; revised 2 December 1999

\begin{abstract}
Dielectric capacities and losses were measured, in the temperature $\left(50-170^{\circ} \mathrm{C}\right)$ and frequency (01-100 kHz range), for undoped and acrylic acid (AA) doped ethyl cellulose (EC) films (thickness about $20 \mathrm{\mu m}$ ) with progressive increase in the concentration of dopant in the polymer matrix. The variation of capacity with temperature is attributed to thermal expansion in the lower temperature region to the orientation of dipolar molecules in the neighbourhood of glass transition temperature $\left(T_{\mathrm{g}}\right)$ and random thermal motion of molecules above $T_{\mathrm{g}}$. The dielectric losses exhibit a broad peak. Doping with AA is found to affect the magnitude and position of the peak. AA is found to have a two-fold action. Firstly, it enhances the chain mobility and secondly, it increases the dielectric loss by forming charge transfer complexes.
\end{abstract}

Keywords. Dielectric capacity; losses; ethyl cellulose; acrylic acid; charge transfer complexes.

\section{Introduction}

The dielectric properties of polymeric films are of direct interest to both the basic studies of electrical conduction through such films, and their application in capacitors for microelectronics (Srivastava and Shrivastava 1981). Extensive observations have been made by several workers to study the molecular motion and the charge-carrier migration in a variety of polar/nonpolar linear polymers containing one, or more than one, active groups in the same monomer unit (Yamafugi 1960; Saito et al 1968; Ishida 1969; Khare et al 1994a). The evaluation of dielectric properties of the insulator film is carried out by measuring simultaneously the capacitance and the dielectric losses of the film over a wide range of frequencies and temperatures (Srivastava and Shrivastava 1981). Although the dielectric properties of a number of polymers have been investigated (Nakagawa and Ishida 1973; Baired and Sengupta 1974; Rastogi and Chopra 1975; Kulshrestha and Srivastava 1980; Mahendru et al 1981; Talwar et al 1985), the molecular orientation behaviour and the associated relaxation mechanisms of the polymers are not fully understood. The dielectric dispersion and absorption are the crucial quantities required in the design of any device. The study of dielectric loss as a function of temperature and frequency, is one of the most convenient and sensitive methods of investigating polymer structure of a polymeric film.

\footnotetext{
*Author for correspondence
}

Considerable attention has been devoted to the problems of the change in the dielectric properties in polymers due to intentional doping with low-molecular-weight compounds (Sasabe et al 1971). The charge-carrier mobility can be greatly influenced by impregnating the polymers with suitable dopants (Mahendru et al 1977). Depending on their chemical structure and the way in which they react with the macromolecular matrix, doping substances decrease the resistivity of the polymer to varying degrees.

In this paper we have studied the dielectric capacity and the dielectric loss behaviour of the undoped ethyl cellulose (EC) and the doped EC with polar acrylic acid; since the introduction of a specific impurity might assist in understanding the relaxation processes more clearly.

Acrylic acid was selected as a doping substance for two reasons: first, it is a monomer, i.e. polymer-making substance, and hence its chances of bringing about a change in the properties of polymer seem to be greater; second, it is a $\alpha$ - $\beta$-unsaturated carbonyl compound containing carboncarbon double bond and carbon-oxygen double bond, and hence the chances of its linking with the polymer also increase (Sinha and Srivastava 1979). A study of dielectric capacity and dielectric-loss behaviour was made over a range of frequencies in undoped and acrylic-acid-doped ethyl cellulose in order to study the effect of the introduced polar impurity on the polarization in the nonpolar matrix.

\section{Experimental}

Commercial ethyl cellulose (EC) used in the present study, having a glass transition temperature $\left(T_{\mathrm{g}}\right), \sim 130^{\circ} \mathrm{C}$ 
and molecular weight, 40,000, was obtained from Glaxo Co., Bombay. The polymer for the preparation of the films, was used as such, without any further purification/ recrystallization. Dopant acrylic acid (AA) used was of Robert Johnson make. The isothermal immersion technique was applied for preparing films of pure and acrylic acid (AA)-doped ethyl cellulose. The solution was prepared in a glass beaker by first dissolving $2 \cdot 1 \mathrm{~g}$ EC in $30 \mathrm{ml}$ chemically pure chloroform at room temperature, and this was continuously stirred with a teflon-coated magnetic stirrer for $30 \mathrm{~min}$. Thereafter, it was stirred and heated to $40^{\circ} \mathrm{C}$ to yield a homogeneous solution. The solution was then immersed in a constant-temperature oil bath. Ultrasonically cleaned vacuum aluminized microscopic glass slides were immersed vertically into the solution for about $10 \mathrm{~min}$. After the deposition of the film, the glass slide was taken out and dried in an oven at $40^{\circ} \mathrm{C}$ for $24 \mathrm{~h}$. This was followed by room-temperature outgassing at $10^{-5}$ torr for a further period of $24 \mathrm{~h}$. The upper electrode was also vacuum deposited on the upper surface of the film to obtain a sandwich configuration. The AA-doped EC films were also prepared by the same technique under identical conditions of taking $2 \cdot 1 \mathrm{~g}$ of EC into well stirred $1 \mathrm{ml}$ of AA that had been thoroughly mixed with $30 \mathrm{ml}$ of chloroform. The doping concentration was changed by varying the amount of AA to be added in different volume of the solvent such that the total volume of solvent and AA remains to be constant. The thickness of the sample was of the order of $20 \mu \mathrm{m}$. The assembly was held in a thermostat, and the temperature was measured using calibrated copper-constantan thermocouple. The capacitance and losses were measured with a Systronics LCR bridge type 921, using a Philips AF generator model GM 2308/90, in the frequency range $01-100 \mathrm{kHz}$, and temperature range $50-180^{\circ} \mathrm{C}$ for undoped and AA-doped EC, in steps of $10^{\circ} \mathrm{C}$.

\section{Results and discussion}

The variation in capacity with temperatures at different frequencies, i.e. $01,05,10,50$ and $100 \mathrm{kHz}$, for undopedethyl cellulose (EC) is shown in figure 1. The capacity decreases first and then increases slightly in the neighbourhood of the glass transition temperature $\left(T_{\mathrm{g}}\right)$, and subsequently decreases continuously. Figure 2 shows the loss variation with temperature at five frequencies (between 1 and $100 \mathrm{kHz}$ ) for undoped EC. A broad losspeak is seen around $130-140^{\circ} \mathrm{C}$ for EC which shifts to lower temperature as the frequency is increased. However, the representative loss thermograms for $\mathrm{EC}$ and acrylic-acid (AA)-doped EC samples, with successive increase in the AA concentrations at $25 \mathrm{kHz}$ are shown in figure 3. Evidently, the position of loss-peak shifts towards lower temperatures and, its magnitude increases progressively with increasing dopant concentration.
Activation energies, for undoped and various dopant concentrations, were calculated from the plots of $\log$ (frequency) and $10^{3} / T$ (results not shown) and were found to decrease with increase of the dopant concentration.

The dielectric behaviour of the polymer is determined by the charge distribution and also by statistical thermal motion of its polar groups. The polarization of a dielectric is contributed by the ionic, electronic, and dipole polarization. The electronic polarization occurs during a very short interval of time (of the order of $10^{-15} \mathrm{sec}$ ) but longer than for electronic polarization, i.e. $10^{-13}-10^{-12} \mathrm{sec}$, required for the process of ionic polarization to set in. Dipole polarization requires a relatively long time compared with that of practically almost inertialess phenomena of deformational polarization, so dielectric constant of nonpolar polymers remains invariable with frequency. In case of polar polymers, the dielectric constant begins to drop at a certain critical frequency. The dipole molecules cannot orient themselves in the lower temperature region. However, owing to thermal expansion, the ratio of the number of molecules to the effective length of the dielectric decreases when the temperature increases. As the temperature increases, the orientation of dipoles is facilitated and this increases the capacity. This behaviour is apparent from the upward trend of the slopes of capacity curves in the neighbourhood of glass transition temperature $\left(T_{\mathrm{g}}\right)$. As the temperature increases further, the chaotic thermal oscillations of molecules are intensified and the degree of order of orientation is diminished (Khare and Chandok 1995). This causes the slopes of capacity curves (figure 1) to fall-off again with temperature.

The dielectric dispersion appearing at high temperatures is generally admitted to be due to the rotatory diffusional motion of the molecules from one quasi-stable position to another around the skeletal bond involving large-scale conformational rearrangement of the main chain, and is known as the primary dispersion region or the $\alpha$-relaxation. The low temperature dielectric dis-

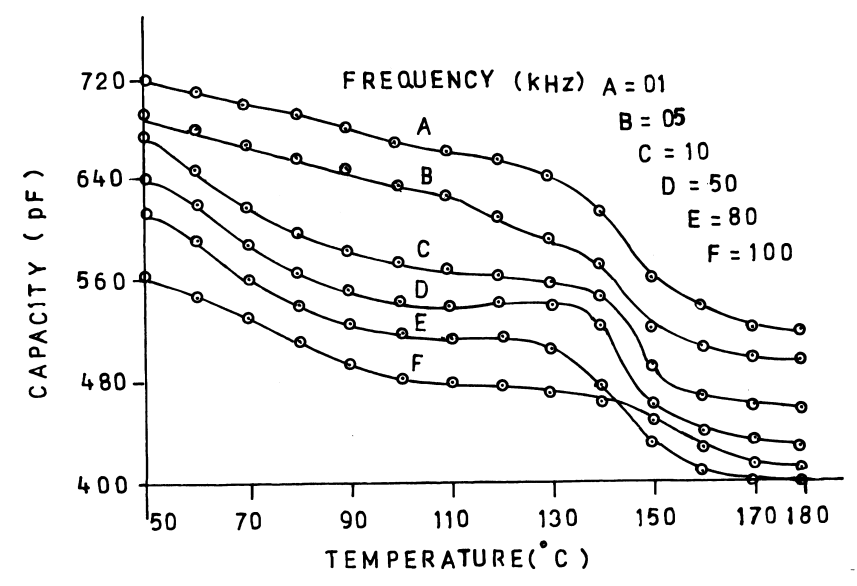

Figure 1. Capacity vs temperature curves at different frequencies: $01,05,10,50,80$ and $100 \mathrm{kHz}$ for EC. 
persion is attributed to the dielectric response of the side groups which are considered to be more mobile or the small displacement of the dipoles near the frozen-in position and known as the secondary dispersion region or $\beta$-relaxation (Mahendru et al 1981).

Thin polymer films are known to be a mixture of amorphous and crystalline regions (Chopra et al 1971). The amorphous regions are the areas in which the chains are irregular and entangled, whereas, in crystalline regions, the chains are regularly folded or orderly arranged. In the crystalline areas, because of the presence of hindering structural units (due to greater density of these regions) the polymeric chains move with a greater difficulty than in the amorphous regions. This hinderance can be assumed to possess a certain potential energy. When the polymer is heated the movements of the main chain sets in, becoming maximum at $T_{\mathrm{g}}$, at which maximum losses occur corresponding to the $\alpha$-relaxation peak of thermally stimulated current spectrum (Khare et al 1992, 1997). This relaxation peak, corresponding to $T_{\mathrm{g}}$, may also be understood by the free volume theory (Fox and Flory 1950) according to which the molecular mobility (and consequently the relaxation time) near $T_{\mathrm{g}}$ depends mainly on the free volume. In the glassy state below $T_{\mathrm{g}}$, the free volume will be frozen in and will remain fixed. As the temperature increases, the glassy state will expand due to normal expansion of all the molecules, which results from the changing vibrational amplitudes of bond distances (Roberts and White 1973). As $T_{\mathrm{g}}$ is reached, in addition to the normal expansion process, there will be an expansion of the free volume itself, which results in a larger expansion of the rubber-like polymer. This yields sufficient room for rotational or translation motion of the molecules to occur at $T_{\mathrm{g}}$, accounting for the maximum dielectric loss at $T_{\mathrm{g}}$ (Talwar et al 1985).

Polarization and depolarization thermograms of EC exhibit two kinds of relaxation processes above the room

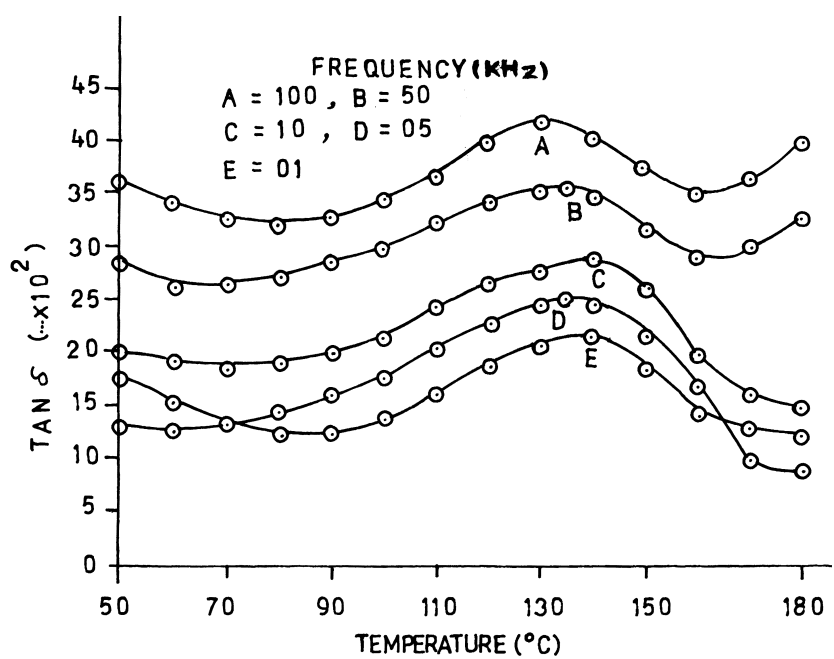

Figure 2. Losses vs temperature curves at different frequencies: $01,05,10,50$ and $100 \mathrm{kHz}$ for EC. temperature. One designated as $\beta$, occurs below $T_{\mathrm{g}}$, and the other designated as $\alpha$, occurs in the neighbourhood of $T_{\mathrm{g}}$ (Khare et al 1992, 1997, 1998b).

Two loss peaks would be expected in the dielectric spectrum of EC, since the dielectric properties are associated with the molecular relaxation modes of a system. In the present case, however, only one peak appears. The position of peak lies between $130-145^{\circ} \mathrm{C}$ over the entire frequency range. Since it is in the higher temperature peak, located in the neighbourhood of $T_{\mathrm{g}}$, it appears to be an $\alpha$-dielectric peak. This higher-temperature peak may be due to deformation accompanied by large changes in the directions or location of the dipoles, which involve a high activation energy. In the deformation process, the dipole orientations will change owing to segmental motion or translation of the main chain. The relaxation time, characteristic of the temperature, is decreased so that $\alpha$-relaxation becomes hardly observable at low temperatures. The location of the peak is comparable with that of $\alpha$ peak in TSC studies (Khare et al 1992, 1997).

The presence of dipolar molecules may also contribute in the process. The dipoles associated with the backbone of a segment of polymer chain will orient with a certain frequency governed by the elastic restoring force which binds the dipoles to their equilibrium positions and the rotational frictional forces exerted by the neighbouring dipoles (Talwar et al 1984). The loss peak observed in the present case is broad and in conventional bridge measurements, distributions of relaxations are contemplated by its width on the frequency scale, whereas, in the TSC studies it is the dependence on the poling conditions which enables one to distinguish between a distributed and a non-distributed relaxation (Khare et al 1998a). In the

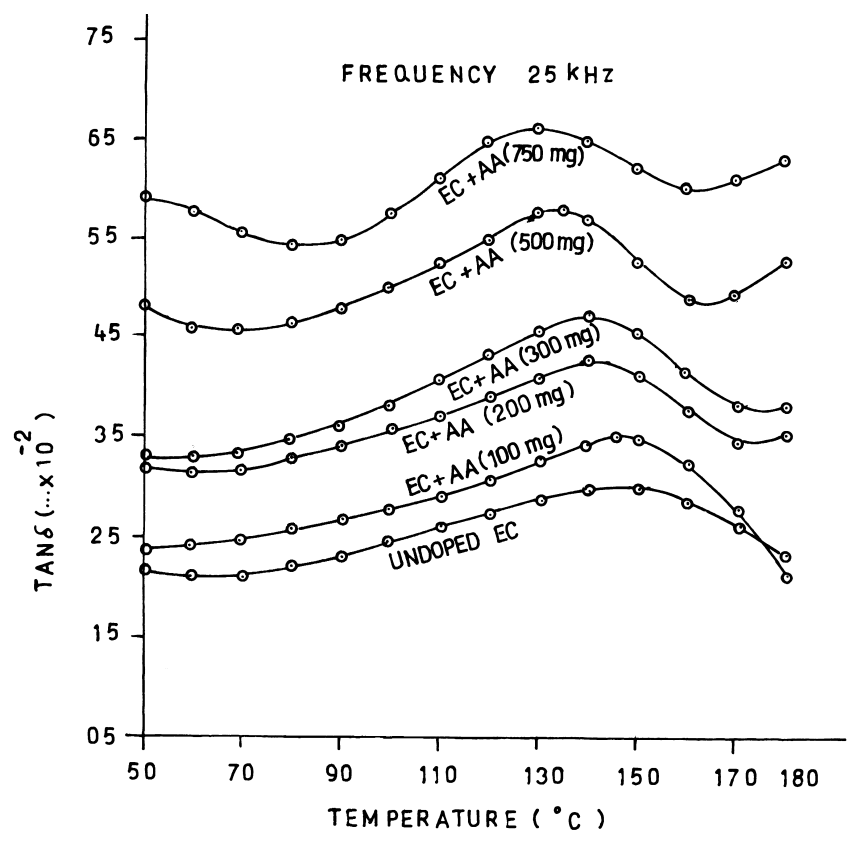

Figure 3. Losses vs temperature curves at $25 \mathrm{kHz}$ for undoped ethyl cellulose and various acrylic acid-doped samples. 
crystalline or ordered polymers, the forces which hold the structural unit together are of a homopolar chemical binding nature and are much stronger than the van der Waal's forces between the molecules in the amorphous phase which vary from region to region. Moreover, the dipolar molecules in the crystalline phase will make discontinuous jumps from one equilibrium position to another and hence contribute to the absorption at the same temperature or frequency, whereas, in the amorphous phase, dipolar molecules should be able to orient from one equilibrium position to another relatively more easily and will contribute to the absorption over a wide frequency or temperature range, since each dipole in the latter case would have slightly different environments and hence would have different intermolecular interaction. If the natural jump frequency of a dipole is $\omega_{0}$, then the dielectric properties of such a dipolar system in an alternating electric field of frequency $\omega$ are described by the complex Debye permittivity. With $\tau=1 / \omega_{0}$, the expression

$$
\Psi(\omega) \propto \frac{1}{(1+i \omega \tau)},
$$

gives a loss relation of the type

$$
\varepsilon^{\prime \prime}=\frac{\left(\varepsilon_{\mathrm{s}}^{\prime}-\varepsilon_{\infty}^{\prime}\right) \omega \tau}{1+\omega^{2} \tau^{2}},
$$

where $\varepsilon_{\mathrm{s}}^{\prime}$ and $\varepsilon_{\infty}^{\prime}$ are the static (low frequency) and high frequency dielectric constants, respectively and $\Psi(\omega)$ is the Debye permittivity. The dielectric medium has a finite rate of response to this excitation, giving rise to a gradual relaxation of energy after a sudden orientation before a final stage is reached. The position in which dipole is to orient lies at a higher energy. After a certain period of time, the dipole from an unrelaxed position relaxes by absorption of energy. With no external field and in thermal equilibrium conditions, detailed balancing ensures that the energy loss incurred every time the dipole relaxes into a new position is fed back into the system via thermal phonons to provide the extra energy for the corresponding upward excitation. Therefore, there is no net loss or gain of energy in the system. The application of an external electric field changes the energy balance, favouring transitions in one direction and opposing those in the other. This results in a net change in polarization, since the dipoles spend more time on average in one position, and thus the dielectric loss (Rastogi and Chopra 1975). In view of the above, the broad loss-peak, which is a characteristic of the disordered system, can be associated with the dipolar molecules involving a superposition of many Debye type relaxations occurring in the amorphous phase. The activation energy associated with loss-peak is attributed to the deformation caused by the molecular motion involving the main chain motion or to the large segments of the main chain. The observations that the peak is broad and it is not symmetrical about its maximum suggest that this peak is due to distributed relaxations in contrast with a single relaxation as deduced by the Debye theory and can be explained as follows. The polymer chain possesses a variety of internal Brownian motions in which segments of the main chain diffuse from relative configuration to another with changing dipole moments. To each such mode of internal rotatory diffusion, involving a coiling or uncoiling of the chain or a segment of the chain, corresponds a characteristic relaxation time. The losses also occur due to electrical conduction (Khare et al 1998b) which increase with the decrease of frequency. The increase in losses at low frequency may be ascribed to interfacial polarization involving ionic movement. With the increase in frequency interfacial polarization decreases and becomes vanishingly small at high frequencies.

The dopant, acrylic acid, has the structural formula<smiles>C=CC(=O)O</smiles>

It is an $\alpha-\beta$-unsaturated carbonyl compound that possesses $\mathrm{C}=\mathrm{C}$ and $\mathrm{C}=\mathrm{O}$ double bond and exhibit the properties of these two functional groups (Talwar et al 1984). Furthermore, AA may be regarded as an electronegative acceptor because of the electron-attracting carbonyl group, the adjacent double bond increasing the tendency of the proton to escape. The double bond present in the doped samples $(\mathrm{C}=\mathrm{C}$ and $\mathrm{C}=\mathrm{O})$ act as deep traps for the carriers, so the energy required to detrap them should be greater. Once the temperature is raised the characteristic of the $\mathrm{C}=\mathrm{O}$ bond, in acting as an electronegative acceptor, increases and the overall behaviour of the acrylic acid molecules is as a more electronegative acceptor, collecting most of the electrons (or ions or both). Ethyl cellulose having a structure $\mathrm{C}-\mathrm{O}-\mathrm{C}$ in its main chain is composed of $\sigma$-donors (Khare et al 1994b). The impregnation of EC structure with AA is likely to form charge transfer complexes (CTC) by donor-acceptor or charge exchange processes between the EC and AA. The formation of CTC is usually characterized by the appearance of a new charge transfer absorption band in the optical absorption spectra of the doped polymer. The UV absorption studies of ethyl cellulose films have indeed shown the appearance of such a band at $284 \mathrm{~nm}$ when doped with acrylic acid, suggesting thereby the formation of CTC between EC and AA. The formation of CTC as a result of AA doping is further confirmed by one observation of the appearance of new peaks in the IR spectra of doped EC samples at 1390 and $1240 \mathrm{~cm}^{-1}$ in addition to the IR peaks already present in the spectrum of undoped samples. The CTC are supposed to create localized states of various depths 
which will lead to trapping sites distributed over a considerably wide energy range. The formation of CTC is considered to reduce the barrier between the trapping sites providing a conducting path through the polymer matrix and would result in the enhancement of its conductivity. Such a phenomena is supposed to decrease the activation energy of the carrier and increase their mobility towards the electrode (Khare and Jain 1997). As discussed earlier, the location of $\alpha$-peak is closely related to the chemical structure, i.e. to the mobility of the main chain. The consistent shifting of the peak position towards lower temperature with increasing AA concentration (figure 3) indicates that the mobility of the EC main chains is increased on the introduction of AA into its structure. The action of AA is simultaneously lowering the $\alpha$-peak temperature and its activation energy is comparable with the action of a plasticizer.

\section{References}

Baired M E and Sengupta C R 1974 Polymer 15608

Chopra K L, Rastogi A C and Malhotra G L 1971 Thin Solid Films 24125

Fox T G and Flory P J 1950 J. Am. Chem. Soc. 702384

Ishida Y 1969 J. Polym. Sci. 71835

Khare P K and Chandok R S 1995 Indian J. Phys. A69 545

Khare P K and Jain S K 1997 Indian J. Pure \& Appl. Phys. 35 408

Khare P K, Surinder P and Srivastava A P 1992 Indian J. Pure \& Appl. Phys. 30165

Khare P K, Gour M S and Ranjeet Singh 1994a Indian J. Phys. A68 545
Khare P K, Keller J M, Gour M S, Ranjeet Singh and Datt S C 1994b Polym. Int. 35337

Khare P K, Kuraria Rajendra and Jain Sandeep 1997 J. Polym. Mater. 14133

Khare P K, Upadhyay J K, Ashish Verma and Paliwal S K 1998a Polym. Int. 47145

Khare P K, Ashish Verma and Paliwal S K 1998b Bull. Mater. Sci. 21207

Kulshrestha Y K and Srivastava A P 1980 Thin Solid Films 71 41

Mahendru P C, Pathak N L, Jain K and Mahendru P 1977 Phys. Status Solidi(a) $\mathbf{4 2} 403$

Mahendru P C, Agrawal J P and Jain K 1981 Indian J. Pure \& Appl. Phys. 19217

Nakagawa K and Ishida Y 1973 J. Polym. Sci. Polym. Phys. 11 1503

Rastogi A C and Chopra K L 1975 Thin Solid Films 27 311

Roberts G E and White E F T 1973 The physics of glossy polymers (ed.) R M Haward (London) p. 1979

Saito S, Sasabe H and Nakajima T 1968 J. Polym. Sci. 6 1297

Sasabe H, Sawamura K, Saito S and Yoda K 1971 Polym. J. 2 518

Sinha H C and Srivastava A P 1979 Indian J. Pure \& Appl. Phys. 17726

Srivastava A P and Shrivastava S K 1981 Indian J. Pure \& Appl. Phys. 19953

Talwar I M, Sinha H C and Srivastava A P 1984 Thin Solid Films 113251

Talwar I M, Sinha H C and Srivastava A P 1985 J. Mater. Sci. L4 448

Yamafugi K 1960 Phys. Soc. Jpn. 152295 